\title{
Coach my Career
}

\author{
Jürg Unger-Köppela ${ }^{a}$, Eva Kaiser ${ }^{b}$, Werner Bauer $^{c}$, Marc Eich $^{d}, K_{\text {arl-Olof Lövblad }}$, Nora Bienz ${ }^{f}$
}

${ }^{\text {a }} \mathrm{FMH} ;{ }^{\mathrm{b}} \mathrm{mfe} ;{ }^{\mathrm{c}} \mathrm{SIWF} ;{ }^{\mathrm{d}} \mathrm{SWIMSA} ;{ }^{e} \mathrm{VLSS} ;{ }^{\dagger} \mathrm{VSAO}$

Die Perspektiven, die sich künftigen Ärztinnen und Ärzten bieten, sind oft nicht vorhersehbar und nicht kontrollierbar. Doch in einem Bereich kann die Ärzteschaft das Steuer in die Hand nehmen: in der Ausbildung, vor allem beim Mentoring des ärztlichen Nachwuchses. Dies gilt besonders für die Planung der Weiterbildung und der beruflichen Entwicklung. Deshalb lancieren die FMH, mfe, das SIWF, die SWIMSA, der VLSS und der VSAO das gemeinsame Projekt «Coach my Career» für junge Ärztinnen und Ärzte.

Früher (und manchmal auch heute noch) standen junge Ärztinnen und Ärzte alleine vor der Entscheidung, wie sie ihre Karriere ausrichten sollten. Ihnen stand kein Ansprechpartner zur Verfügung, an den sie sich mit ihren Fragen wenden konnten: Wie bilde ich mich zur Kinderärztin aus, wie werde ich Forscher, wie gehe ich vor, wenn ich Spitalärztin oder ordentlicher Professor in einem bestimmten Bereich werden möchte? Abgesehen von wenigen Ausnahmen erfolgte die berufliche Reise von angehenden Ärztinnen und Ärzten aufs Geratewohl, und verschiedene Variablen wurden nicht berücksichtigt. Aus diesem Grund haben sich die FMH (Departement Stationäre Versorgung und Tarife), Verband Schweizerischer Assistenz- und Oberärztinnen und -ärzte VSAO, Verein der Leitenden Spitalärzte der Schweiz VLSS, mfe Haus- und Kinderärzte Schweiz und Swiss Medical Students' Association SWIMSA zusammengetan und ein neues Projekt aufgegleist, bei dem sie auch vom Schweizerischen Institut für ärztliche Weiter- und Fortbildung SIWF unterstützt werden. Unter dem Titel «Coach my Career» sollen erfahrene Kräfte aus der Ärzteschaft dem Nachwuchs helfen, einen guten Weg in die berufliche Zukunft zu finden, bei der auch die private Situation mitberücksichtigt wird. Deshalb richtet sich das Projekt vor allem an zwei Gruppen: Medizinstudierende im letzten Studienjahr sowie junge Assistenz- und Oberärztinnen und -ärzte, die vor wichtigen beruflichen Weichenstellungen stehen.

\section{Unterstützung und Beratung für den ärztlichen Nachwuchs}

Das wirtschaftliche und soziale Umfeld, in dem Ärztinnen und Ärzte tätig sind, ist von tiefgreifenden Veränderungen geprägt. Laufend eröffnen sich neue beruf- liche Möglichkeiten, doch gleichzeitig ändern sich oft die Curricula oder werden gar aufgehoben. Angesichts dieser Veränderungen und Herausforderungen ist es für junge Ärztinnen und Ärzte oft schwierig, ihren Weg durch den beruflichen Dschungel zu finden. Zu Beginn ihres Berufslebens stehen sie mehrmals vor wichtigen Entscheidungen. So stellen sich gegen Ende des Studiums Fragen zur allgemeinen Ausrichtung der Berufstätigkeit: Werde ich Allgemeinmedizinerin oder Facharzt, welche der 45 Fachdisziplinen wähle ich, wende ich mich der Forschung oder der Industrie zu? Später folgen weitere wichtige Entscheidungen bezüglich der Eröffnung einer eigenen Praxis oder einer Spital- oder Hochschulkarriere. Dazu kommt die Herausforderung, die Berufstätigkeit mit einer angemessenen Lebensqualität im privaten und familiären Bereich $\mathrm{zu}$ vereinbaren. Angesichts von wirtschaftlichen Aussichten, die weniger rosig sind als auch schon, erscheint es uns wichtig, das Interesse der jungen Ärztinnen und Ärzte an ihrer beruflichen Zukunft aufrechtzuerhalten. Deshalb möchten wir ihnen die Möglichkeit bieten, sich von älteren Kolleginnen und Kollegen unterstützen und beraten zu lassen.

Das Projekt startet zunächst in der Deutschschweiz und soll anschliessend auf die ganze Schweiz ausgedehnt werden. Für die Medizinstudentinnen und Medizinstudenten werden unter der Federführung der SWIMSA Informationsveranstaltungen in den verschiedenen medizinischen Fakultäten durchgeführt. Für die Assistenz- und Oberärztinnen und -ärzte erfolgt ein Coaching der einzelnen Kandidatinnen und Kandidaten jeweils durch zwei erfahrene Kolleginnen oder Kollegen.

Das Projekt ergänzt die Coachingprojekte, die an den medizinischen Fakultäten bereits bestehen oder zurzeit aufgebaut werden. Es stellt keine Hilfestellung zur 
Erfüllung der Kriterien für eine bestimmte Weiterbildung dar, sondern bietet den jungen Ärztinnen und Ärzten vielmehr eine Orientierungshilfe hinsichtlich ihrer verschiedenen Karrierepläne.

\section{Win-win-Situation für alle Beteiligten}

Für das Mentoring sollen sowohl pensionierte Chefoder Kaderärztinnen und -ärzte als auch jüngere, noch berufstätige Personen sowie gut etablierte Hausärztinnen und -ärzte gewonnen werden. Die künftigen Mentorinnen und Mentoren sollten über ausgewiesene Erfahrung in der Weiterbildung verfügen. Das Ziel besteht darin, dass ihre Erfahrungen weiterhin genutzt und zugunsten der jüngeren Kolleginnen und Kollegen eingesetzt werden können. Die Erfahrungen der Mentorinnen und Mentoren sollen der Ärzteschaft erhalten bleiben und nach der Pensionierung nicht verloren gehen. Damit schafft das Projekt eine Win-winSituation, die allen beteiligten Berufskategorien Vorteile bringt. Neben ihrer persönlichen Erfahrung sollen die Mentorinnen und Mentoren auch über ein ausgedehntes fachliches Netzwerk verfügen. Es werden engagierte Coaches gesucht, die daran interessiert sind, diese Aufgabe zu übernehmen und eine aktive Rolle zu spielen. Ihre Sozialkompetenz motiviert sie dazu, Jüngere zu coachen. Dabei ist es nicht unbedingt notwendig, dass die Mentorinnen und Mentoren über die gleiche Ausbildung verfügen wie die gecoachten Ärztinnen und Ärzte. Denn es ist im System vorgesehen, dass den Mentees ein Coach-Tandem gegenübersitzt: Ein Coach vertritt das gewünschte Fach des Mentee, während der zweite Coach aus einem anderen Fach kommt, um so die Diskussion zu bereichern. Dies ermöglicht ausgewogenere Gespräche und gewährleistet neben einem gezielten Mentoring auch ein allgemeineres Coaching.

Die Rolle der jüngeren Mentorinnen und Mentoren wird auch darin bestehen, den Mentees, die dies wünschen, eine Person zur Seite zu stellen, die altersmässig näher bei ihnen liegt und ihre persönlichen Erfahrungen eher teilt.

Generell werden die Mentees einer der drei bereits erwähnten Kategorien angehören:

- Medizinstudierende vor dem Abschluss ihres Studiums, die eine Orientierungshilfe für die Wahl ihrer Weiterbildungsstätte und des Fachgebiets entsprechend ihren Bedürfnissen benötigen;

- Assistenzärztinnen und -ärzte, die erfahren möchten, wie sie ihre Karriere gestalten und so mit dem Privat- und Familienleben vereinbaren können, dass ihre Weiterbildung unter optimalen Bedingungen ablaufen kann;
- Ärztinnen und Ärzte, die ihre Weiterbildung abgeschlossen haben und als Oberärztinnen und -ärzte tätig sind, die entweder wissen möchten, wie sie sich selbständig machen oder wie sie in ihrem Fachgebiet eine Hochschullaufbahn mit allen damit verbundenen Anforderungen einschlagen können (Praktikum im Ausland, Habilitation, Beförderungen im Spital und an der Universität).

Die Häufigkeit der Treffen zwischen Mentoren und Mentee wird unterschiedlich sein.

Einzigartig ist dieses Projekt unter anderem, weil es die verschiedenen Gruppierungen der Ärzteschaft unter einem Dach vereint für ein gemeinsames Ziel: die Unterstützung unserer jungen Ärztinnen und Ärzte im Hinblick auf ihre berufliche Zukunft. Dieses verbindende Projekt bringt die Mentees (Mitglieder der SWIMSA oder des VSAO) mit den Mentorinnen und Mentoren (FMH, VLSS, mfe und zum Teil VSAO) zusammen. Das Sekretariat und den Support übernimmt aktuell der VLSS. Der Verband übernimmt nach den Wünschen der Mentees, die bei der Anmeldung erfasst werden, das Zuteilen der Mentorinnen und Mentoren. Der Schweizer Ärzteschaft bietet dieses Projekt eine einmalige Chance: So können die Kompetenzen der pensionierten Kaderärztinnen und -ärzte weiterhin genutzt werden, und zugleich kann dem ärztlichen Nachwuchs in einem medizinischen und wirtschaftlichen Umfeld, das sich laufend verändert, ein Mentoring angeboten werden. Gerne rufen wir hier ehemalige Chef- und Kaderärztinnen und -ärzte sowie erfahrene Praktikerinnen und Praktiker auf, unsere künftigen Ärztegenerationen zu unterstützen und sie bei ihren manchmal schwierigen Entscheidungen zu begleiten. Künftige Coaches, die sich für die Aufgabe interessieren, können sich direkt beim Sekretariat des VLSS melden. Sobald eine Datenbank mit potentiellen Mentorinnen und Mentoren aufgebaut ist, die den jungen Ärztinnen und Ärzten ein breites Spektrum von Fachgebieten und Möglichkeiten bieten, wird das Projekt gestartet. Als erster Schritt erfolgt die Abstimmung unter den Coaches, und zugleich werden die Mentees eingeladen werden, sich für ein Coaching anzumelden.

Wir freuen uns, wenn sich möglichst viele engagierte Mentorinnen und Mentoren beim Sekretariat des VLSS melden. Sobald sich genügend Mentoren gemeldet haben, werden sie zu einem Mentorentreffen eingeladen, und zugleich werden die Mentees eingeladen, sich anzumelden.

Adresse des VLSS:

Verein der Leitenden Spitalärzte der Schweiz VLSS,

Sekretariat, Postgasse 19, Postfach, $\mathrm{CH}-3000$ Bern 8,

Tel. 03133090 01, Fax 03133090 03, info[at]vlss.ch, www.vlss.ch 